\title{
APPLICATION OF DM METHODS FOR PROBLEMS WITH PARTIAL DIFFERENTIAL EQUATIONS
}

\author{
T. CİRULIS and O. LIETUVIETIS
}

Institute of Mathematics of Latvian Academy of Sciences and University of Latvia

Akadēmijas laukums 1, Rīga LV-1524, Latvia

E-mail: cirulis@lanet.lv, ojarsl@lanet.lv

Received October 1, 2002

\begin{abstract}
Two variants of applications of the Degenerate Matrix Method for solving problems with PDE are considered. Solutions of the simple testing problem and of one more complicated nonlinear problem with PDE of the fifth order are presented as examples.
\end{abstract}

Key words: numerical algorithms, partial differential equations.

\section{INTRODUCTION}

Degenerate matrix methods (DM methods) appear to be very suitable and efficient for numerical solution of initial value problems of Ordinary Differential Equations (ODE). It is especially noticeable if the nodes on the mold are chosen as zeroes of certain classical orthogonal polynomials ensuring the nonsaturatedness of approximations of functions. Such methods for ODE were discussed in $[2 ; 3 ; 4 ; 5]$. Here we will consider how DM methods can be applied to Partial Differential Equations (PDE) accenting at first the cases when nodes of the mold are distributed as zeroes of certain classical orthogonal polynomials, for example, Chebyshev polynomials of the second kind. In Section 2 we will consider a general scheme of the method. In Section 3 we will compare the precise solution for some test problem with numerical solutions obtained by means of proposed approximate methods. In Section 4 we will analyze the numerical solution obtained by means of DM methods for sufficiently complicated nonlinear problem of PDE. This problem was given 
in [1].

\section{GENERAL SCHEME OF APPLICATIONS OF DM METHODS FOR PDE}

In order to simplify the principal concepts of the DM methods for PDE we consider the computing scheme for the following simplified problem:

$$
\begin{aligned}
& \frac{\partial u}{\partial t}=A\left(t, x, u, \frac{\partial u}{\partial x}\right) \frac{\partial^{2} u}{\partial x^{2}}+B\left(t, x, u, \frac{\partial u}{\partial x}\right),\left.u\right|_{t=0}=f(x) \\
& \left.G_{1}\left(t, u, \frac{\partial u}{\partial x}\right)\right|_{x=a}=0,\left.\quad G_{2}\left(t, u, \frac{\partial u}{\partial x}\right)\right|_{x=b}=0
\end{aligned}
$$

where $A, B, G_{1}, G_{2}$ are given functions of their arguments. We propose two variants for solving the problem $(2.1)-(2.2)$.

Variant A. $1^{\circ}$. Let $N+2$ nodes $a=x_{0}<x_{1}<\ldots<x_{N}<x_{N+1}=b$ be given in the closed interval $[a, b]$ which can be reduced by substitution $x=a+0.5(s+1)(b-a)$ to $[-1,1]$. Then for each $x_{k}, k=0,1, \ldots, N+1$, it is possible to determine uniquely $s_{k}=\left(2 x_{k}-a-b\right) /(b-a) \in[-1,1]$. The choice of $x_{k}$ contains usually the interior nodes $s_{k}, k=1,2, \ldots, N$ as zeroes of classical orthogonal polynomials. In such way a nonsaturatedness of approximations of functions with respect to $x$ can be reached.

$2^{\circ}$. The equation (2.1) with respect to $x$ is contracted to interior nodes $x_{1}, \ldots, x_{N}$. Then the vectors of derivatives

$$
\left(\frac{\partial^{m}}{\partial x^{m}} u\left(t, x_{1}\right), \frac{\partial^{m}}{\partial x^{m}} u\left(t, x_{2}\right), \ldots, \frac{\partial^{m}}{\partial x^{m}} u\left(t, x_{N}\right)\right), \quad m=1,2,
$$

are replaced by means of the matrices for derivatives according to the chosen nodes in the closed interval $[a, b]$ analogically as in the methods for ODE. We denote

$$
\vec{U}_{x}^{(m)}:=\left(\frac{\partial^{m}}{\partial x^{m}} u\left(t, x_{0}\right), \frac{\partial^{m}}{\partial x^{m}} u\left(t, x_{1}\right), \ldots, \frac{\partial^{m}}{\partial x^{m}} u\left(t, x_{N+1}\right)\right),
$$

where $m=0,1,2, \vec{U}:=\vec{U}_{x}^{(0)}$. These vectors have values of $\frac{\partial^{m} u}{\partial x^{m}}$ in all chosen nodes at the closed interval $[a, b]$ as components and can be approximately calculated by

$$
\vec{U}_{x}^{(m)}=\vec{U}\left(\mathbf{A}_{x}^{\top}\right)^{m}, m=1,2 .
$$

Here $\mathbf{A}_{x}$ is the $(N+2) \times(N+2)$ matrix for derivatives according to nodes $x_{0}, x_{1}, \ldots, x_{N+1}$ and elements $a_{k, j}$ of this matrix can be computed by the simple formulas [3].

$$
a_{k, j}=0.5(b-a) \frac{p^{\prime}\left(s_{k}\right)}{\left(s_{k}-s_{j}\right) p^{\prime}\left(s_{j}\right)}, \text { if } k \neq j
$$




$$
a_{k, k}=0.5(b-a) \frac{p^{\prime \prime}\left(s_{k}\right)}{2 p^{\prime}\left(s_{k}\right)}
$$

were $j, k=0,1, \ldots, N+1,-1=s_{0}<s_{1}<\ldots<s_{N}<s_{N+1}=1$ are nodes in the standard interval $[-1,1]$ and

$$
p(s)=\prod_{i=0}^{N+1}\left(s-s_{i}\right)
$$

The matrix $\mathbf{A}_{x}$ is degenerate since it has the rank equal to $N+1$. Replacing by (2.5) all derivatives according to $x$ both in the interior points $x_{1}, x_{2}, \ldots, x_{N}$ of contracted equation (2.1) and in the boundary conditions (2.2) we obtain using $u_{k}:=u\left(t, x_{k}\right)$ the following problem for Differential-Algebraic Equations (DAE).

$$
\frac{d u_{k}}{d t}=A\left(t, x_{k}, u_{k}, \sum_{j=0}^{N+1} a_{k, j} u_{j}\right) \sum_{j=0}^{N+1} a_{k, j}^{(2)} u_{j}+B\left(t, x_{k}, u_{k}, \sum_{i=0}^{N+1} a_{k, i} u_{i}\right)
$$

where $k=1,2, \ldots, N$, and $a_{k, j}^{(2)}$ are elements of the matrix $\mathbf{A}_{x}^{2}$,

$$
\begin{aligned}
& G_{1}\left(t, u_{0}, \sum_{j=0}^{N+1} a_{0, j} u_{j}\right)=0, \\
& G_{2}\left(t, u_{N+1}, \sum_{j=0}^{N+1} a_{N+1, j} u_{j}\right)=0 .
\end{aligned}
$$

This problem $(2.8)-(2.10)$ contains the system of $N$ differential equations and two algebraic equations. Solving of such problems for DAE is considered in [6]. The problem $(2.8)-(2.10)$ can be presented also in the following vector-matrix form. Let

$$
v_{k}:=A\left(t, x_{k}, u_{k}, \sum_{j=0}^{N+1} a_{k, j} u_{j}\right), w_{k}:=B\left(t, x_{k}, u_{k}, \sum_{j=0}^{N+1} a_{k, j} u_{j}\right)
$$

for $k=0,1, \ldots, N+1$ and $\vec{W}:=\left(w_{0}, w_{1}, \ldots w_{N+1}\right)$. Eliminating the first and last scalar equations in

$$
\frac{d \vec{U}}{d t}=\vec{U}\left(\mathbf{A}_{x}^{\top}\right)^{2} \operatorname{diag}\left(v_{k}\right)+\vec{W}
$$

and adjoining (2.9) and (2.10) to obtained $N$ equations we have the problem equivalent with $(2.8)-(2.10)$. 
$3^{\circ}$. If this $\mathrm{ADE}$ problem (2.8) - (2.10) is of index 1 then the values of function $u$ at endpoints $x=a, x=b$ or $u_{0}, u_{N+1}$ can be excluded. In this case we obtain the problem for ODE with respect to $u\left(t, x_{k}\right), k=1,2, \ldots, N$. Thus, now the method is finished using the straight line method. DM methods often are appropriate for solving the received ODE guaranteeing a high accuracy.

Variant B. This case differs from the variant $A$ only in that the matrix equation (2.12) obtained in point $3^{\circ}$ has been multiplied one or several times by the pseudo-inverse matrix $\mathbf{B}_{x}^{\top}$ of the matrix for derivatives $\mathbf{A}_{x}^{\top}$. Those matrices are considered in [3]. They can be defined by

$$
\mathbf{B}_{x} \mathbf{A}_{x}=\mathbf{E}-\mathbf{I} \text { or } \mathbf{A}_{x}^{\top} \mathbf{B}_{x}^{\top}=\mathbf{E}-\mathbf{I}^{\top}
$$

where $\mathbf{E}$ is the identity matrix and $(N+2) \times(N+2)$ matrix $\mathbf{I}$ is chosen according to the equality

$$
\operatorname{rank}(\mathbf{E}-\mathbf{I})=N+1
$$

Often we choose as $\mathbf{I}$ some matrix $\mathbf{I}_{m}$ which has all elements equal to zero except the $m$-th column consisting of 1 's, or a linear combination

$$
\mathbf{I}=\alpha_{1} \mathbf{I}_{1}+\alpha_{2} \mathbf{I}_{2}+\ldots+\alpha_{m} \mathbf{I}_{m}
$$

with

$$
\alpha_{1}+\alpha_{2}+\ldots+\alpha_{m}=1
$$

We note that the equality $\mathbf{I}=\mathbf{I}_{1}$ can be usually applied for ODE.

An application of pseudo-inverses matrices is appropriate if the equation (2.1) can be represented in the form

$$
\frac{\partial u}{\partial t}=\frac{\partial}{\partial x}\left(A_{1}(t, x, u) \frac{\partial u}{\partial x}\right)+B_{1}\left(t, x, u, \frac{\partial u}{\partial x}\right)
$$

For example, such representation is possible if the function $A$ in (2.1) does not depend on $u_{x}^{\prime}$.

The considered solving schemes can be also used if the problem $(2.1)-(2.2)$ contains:

1) the derivative $\frac{\partial^{2} u}{\partial t^{2}}$ instead of $\frac{\partial u}{\partial t}$

2) nonlocal boundary conditions instead of (2.2);

3 ) derivatives of higher order than 2 with respect to $x$. 


\section{THE SIMPLE TEST PROBLEM}

In order to determine the effectiveness of the schemes presented in the previous Section we consider as an example the problem

$$
\begin{gathered}
\frac{\partial u}{\partial t}=a^{2} \frac{\partial^{2} u}{\partial x^{2}}+c t \\
\left.\frac{\partial u}{\partial x}\right|_{x=-1}=\left.\frac{\partial u}{\partial x}\right|_{x=1}=0 \\
\left.u\right|_{t=0}=f(x)=c_{0}-c_{1} \sin \frac{\pi x}{2}+c_{2} \cos (2 \pi x) .
\end{gathered}
$$

Here $a, c, c_{0}, c_{1}, c_{2} \in \mathbb{R}$ are given constants. The exact solution of the problem is given by

$$
u(t, x)=\frac{c t^{2}}{2}+c_{0}-c_{1} \sin \frac{\pi x}{2} \exp \left(-\frac{a^{2} \pi^{2} t}{4}\right)+c_{2} \cos (2 \pi x) \exp \left(-4 a^{2} \pi^{2} t\right) .
$$

We will consider here both variants of the method.

Variant A. Choosing $N+2$ nodes

$$
-1=x_{0}<x_{1}<\ldots<x_{N}<x_{N+1}=1,
$$

where $x_{1}, x_{2}, \ldots, x_{N}$ are, for example, zeroes of Chebyshev polynomials of the second kind and applying the $(N+2) \times(N+2)$ matrix $\mathbf{A}_{x}=\left(a_{k, j}\right)$ for derivatives we discretize the equation (3.1) with respect to $x$ :

$$
\frac{d \vec{U}}{d t}=a^{2} \vec{U}\left(\mathbf{A}_{x}^{\top}\right)^{2}+c t \overrightarrow{1}
$$

Notation from Section 2 are used also here and $\overrightarrow{1}:=(1,1, \ldots, 1)$. Now we must use the vector equation (3.4) without first and last components, but $u_{0}$ and $u_{N+1}$ must be eliminated using discretized boundary conditions (3.1) that is, two linear algebraic equations:

$$
\begin{aligned}
& \sum_{j=0}^{N+1} a_{0, j} u_{j}=0 \\
& \sum_{j=0}^{N+1} a_{N+1, j} u_{j}=0 .
\end{aligned}
$$

After that we obtain the system of ODE for $u_{k}=u\left(t, x_{k}\right), k=1,2, \ldots, N$ :

$$
\frac{d \vec{u}}{d t}=a^{2} \vec{u} \mathbf{S}+c t \overrightarrow{1} .
$$

Here $\vec{u}=\left(u_{1}, u_{2}, \ldots, u_{N}\right)$, and elements of $N \times N$ matrix $\mathbf{S}$ are in the form

$$
s_{k, j}=a_{k, j}^{(2)}+a_{k, 0}^{(2)} \beta_{j}+a_{k, N+1}^{(2)} \gamma_{j}, k, j=1,2, \ldots, N,
$$


where $a_{k, j}^{(2)}$ are elements of the matrix $\left(\mathbf{A}_{x}\right)^{2}$,

$$
\begin{aligned}
& \beta_{j}=\frac{1}{d}\left(a_{0, N+1} a_{N+1, j}-a_{N+1, N+1} a_{0, j}\right), \\
& \gamma_{j}=\frac{1}{d}\left(a_{N+1,0} a_{0, j}-a_{0,0} a_{N+1, j}\right), \\
& d=a_{0,0} a_{N+1, N+1}-a_{0, N+1} a_{N+1,0} .
\end{aligned}
$$

The values of $f(x)$ on nodes $x_{1}, x_{2}, \ldots, x_{N}$ are chosen as the initial values for ODE (3.6). This problem was also solved using the DM method with nodes

$$
0=t_{0}<t_{1}<\ldots<t_{M}<t_{M+1}=h
$$

for $M=2$ and different step sizes $h$. Such methods were in detail analyzed in $[3 ; 4]$ and are not considered here.

Variant B. Now after obtaining (3.5) we define the pseudo-inverse matrix $\mathbf{B}_{x}=\left(b_{k, j}\right)$ for $\mathbf{A}_{x}$ by equality

$$
\mathbf{B}_{x} \mathbf{A}_{x}=\mathbf{E}-\mathbf{I}
$$

where $\mathbf{I}=\left(\mathbf{I}_{1}+\mathbf{I}_{N+2}\right) / 2$ is one of matrices (2.15). Multiplying twice (3.4) from the right side by $\left(\mathbf{B}_{x}^{\top}\right)^{2}$ and taking in account boundary conditions in (3.1) leads to

$$
\frac{d \vec{U}}{d t}\left(\mathbf{B}_{x}^{\top}\right)^{2}=a^{2} \vec{U}-\frac{a^{2}}{2}\left(u_{0}+u_{N+1}\right) \overrightarrow{1}=c t \overrightarrow{1}\left(\mathbf{B}_{x}^{\top}\right)^{2} .
$$

We must use the equality (3.9) by analogy with (3.4) without first and last components. The values $u_{0}, u_{N+1}, \frac{d u_{0}}{d t}$ and $\frac{d u_{N+1}}{d t}$ on the boundaries $x= \pm 1$ also must be eliminated from (3.9). For $u_{0}$ and $u_{N+1}$ we have already the equations (3.5), but for derivatives - the similar relations

$$
\frac{d u_{0}}{d t}=\sum_{j=1}^{N} \beta_{j} \frac{d u_{j}}{d t}, \quad \frac{d u_{N+1}}{d t}=\sum_{j=1}^{N} \gamma_{j} \frac{d u_{j}}{d t}
$$

which follow from (3.5) and boundary conditions in (3.1). Finally we have the following implicit system of ODE for $u_{i}=u\left(t, x_{i}\right), i=1,2, \ldots, N$ :

$$
\frac{d \vec{u}}{d t} \mathbf{P}=a^{2} \vec{u}-\frac{a^{2}}{2} \overrightarrow{1} \sum_{j=1}^{N}\left(\beta_{j}+\gamma_{j}\right) u_{j}+c t \vec{w}
$$

where $\mathbf{P}$ is the $N \times N$ matrix with elements

$$
\left\{\begin{array}{l}
p_{k, j}=b_{k, j}^{(2)}+\beta_{j} b_{k, 0}^{(2)}+\gamma_{j} b_{k, N+1}^{(2)} ; \\
\vec{w}=\frac{1}{2}\left(x_{1}^{2}-1, x_{2}^{2}-1, \ldots, x_{N}^{2}-1\right)
\end{array}\right.
$$


Table 1.

Errors of numerical results for the test problem

\begin{tabular}{lllll}
\hline \multirow{2}{*}{$\mathrm{N}$} & \multicolumn{3}{c}{ Variant A } & \multicolumn{2}{c}{ Variant B } \\
\cline { 2 - 5 } & $h$ & $\operatorname{err}_{1}$ & $h$ & err $_{2}$ \\
\hline 5 & $1 / 50$ & $3,7 \cdot 10^{-1}$ & $1 / 100$ & $2,6 \cdot 10^{-2}$ \\
7 & $1 / 100$ & $1,9.10^{-2}$ & $1 / 200$ & $9,6 \cdot 10^{-4}$ \\
9 & $1 / 250$ & $4,1.10^{-3}$ & $1 / 400$ & $4,8 \cdot 10^{-5}$ \\
11 & $1 / 500$ & $2,9.10^{-4}$ & $1 / 800$ & $1,7 \cdot 10^{-6}$ \\
13 & $1 / 1000$ & $1,5 \cdot 10^{-5}$ & $1 / 1500$ & $5,1.10^{-8}$ \\
15 & $1 / 1500$ & $6,1.10^{-7}$ & $1 / 3000$ & $1,2.10^{-9}$ \\
\hline
\end{tabular}

$b_{k, j}^{(2)}$ are elements of $\left(\mathbf{B}_{x}\right)^{2}$. After multiplying from right side by $\mathbf{P}^{-1}$ we can use then also the DM method for solving (3.11) in analogy with the Variant 1.

Numerical experiments. The best numerical results for problem (3.1) (3.2) were achieved choosing grid points for $x$ as zeroes of Chebyshev polynomials of the second kind. The magnitudes of the global errors of both variants for $t=1$ and values of constants $a=2, c=50, c_{0}=1, c_{1}=2, c_{2}=3$ are given in the Table 1 . In calculations we use $M=2$, different $N$ and different step sizes $h$ for $t$. Here we denote

$$
\begin{aligned}
& \operatorname{err}_{1}=\max _{k}\left|u\left(1, x_{k}\right)-\tilde{u}\left(1, x_{k}\right)\right|, \\
& \operatorname{err}_{2}=\max _{k}\left|u\left(1, x_{k}\right)-\hat{u}\left(1, x_{k}\right)\right|,
\end{aligned}
$$

where $u\left(1, x_{k}\right)$ are exact values, calculated by (3.3), and $\tilde{u}, \hat{u}$ - corresponding rough values.

From Table 1 it is obvious that the results obtained using pseudo-inverse matrices (Variant B) are more accurate than the Variant A.

\section{NUMERICAL SOLUTION OF THE NONLINEAR PROBLEM OF PDE}

Here we consider a mathematical problem which is proposed as the model of dynamics of an elongated droplet under the action of a low frequency rotating magnetic field [1].

Mathematical formulation of the problem is the following. It is necessary to solve the equation

$$
\frac{\partial}{\partial t}\left(u+\varepsilon \frac{\partial^{4} u}{\partial x^{4}}\right)=\frac{\partial^{2}}{\partial x^{2}}(\sin 2 u+\alpha u)+\omega
$$

with initial values

$$
\left.u\right|_{t=0}=f(x)
$$


and boundary conditions:

$$
\begin{aligned}
& \left.\frac{\partial u}{\partial x}\right|_{x=-1}=c_{1},\left.\frac{\partial u}{\partial x}\right|_{x=1}=c_{2}, \\
& \left.\varepsilon \frac{\partial^{3} u}{\partial x^{2} \partial t}\right|_{x= \pm 1}=\left.\sin 2 u\right|_{x= \pm 1} .
\end{aligned}
$$

Here $\varepsilon=10^{-2} \div 10^{-4} ; \omega=3 \div 5 ; 0<\alpha<2 ; c_{1}$ and $c_{2}$ are given constants. If $c_{1}=-c_{2}$ and $f(x)$ is even function of $x \in[-1,1]$, then the solution is also the even function with respect to $x$. We will consider only this symmetric case taking $c_{1}=-c_{2}=c$. Let $x_{0}, x_{1}, \ldots, x_{N}, x_{N+1}$ be nodes in $[-1,1]$ and

$$
u\left(t, x_{k}\right):=u_{k}(t), \quad \vec{U}:=\left(u_{0}(t), u_{1}(t), \ldots, u_{N+1}(t)\right) .
$$

Discretization according to $x$ by means of matrices for derivatives leads to the equation

$$
\frac{d \vec{U}}{d t}\left(\mathbf{E}+\varepsilon\left(\mathbf{A}_{x}^{\top}\right)^{4}\right)=(\sin 2 \vec{U}+\alpha \vec{U})\left(\mathbf{A}_{x}^{\top}\right)^{2}+\omega \overrightarrow{1} .
$$

It can be reduced to a more simple system for ODE by multiplying (4.5) from the right side two times with pseudo-inverse matrix $\mathbf{B}_{x}^{\top}$ defined by (3.8), and eliminating $u_{0}(t)$ and $u_{N+1}(t)$ by means of boundary conditions (4.3) (see, the variant $B$ of Section 3). After some simple but long computations we obtain the following system of ODE.

$$
\begin{gathered}
\sum_{j=1}^{N} s_{k, j} \frac{d u_{j}(t)}{d t}=F_{k}\left(x_{1}, \ldots, x_{N}, u_{1}(t), \ldots, u_{N+1}(t)\right), \\
u_{k}(0)=f\left(x_{k}\right), k=1,2, \ldots, N
\end{gathered}
$$

where

$$
\begin{aligned}
& s_{k, j}=\alpha_{k, j}+\varepsilon \sigma_{k, j}-\varepsilon x_{k} \theta_{j}, \quad \alpha_{k, j}=b_{k, j}^{(2)}+b_{k, 0}^{(2)} \beta_{j}+b_{k, N+1}^{(2)} \gamma_{j}, \\
& \sigma_{k, j}=a_{k, j}^{(2)}+a_{k, j}^{(2)} \beta_{j}+a_{k, N+1}^{(2)} \gamma_{j}, \theta_{j}=\frac{1}{2} \sum_{i=1}^{N}\left(a_{0, i}+a_{N+1, i}\right) \sigma_{i, j}, \\
& F_{k}=\sin 2 u_{k}(t)+\alpha u_{k}(t)+\frac{\omega}{2}\left(x_{k}^{2}-1\right)- \\
&-\frac{\alpha}{2}\left[\frac{c}{d}\left(a_{N+1, N+1}+a_{0, N+1}-a_{0,0}-a_{N+1,0}\right)+\sum_{j=1}^{N}\left(\beta_{j}+\gamma_{j}\right) u_{j}(t)\right],
\end{aligned}
$$

and $a_{k, j}, a_{k, j}^{(2)}, b_{k, j}^{(2)}, d, \beta_{j}, \gamma_{j}$ are the same as in Section 3. 


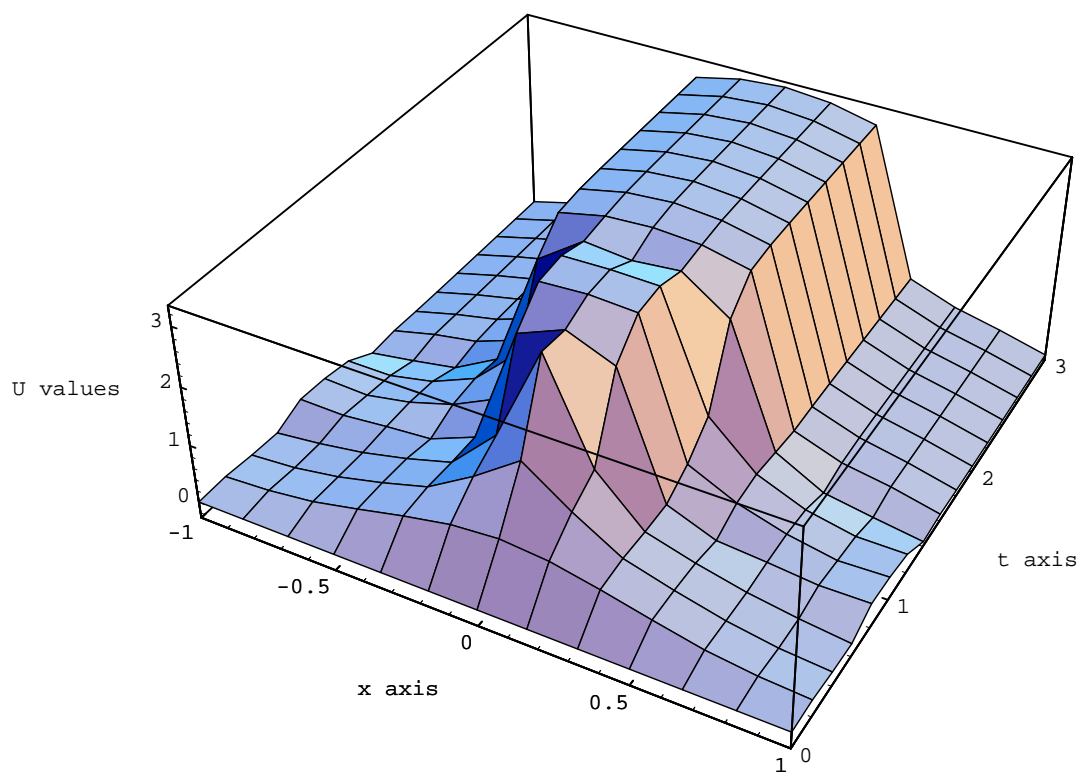

Figure 1. The sample of numerical solution

The problem (4.1) - (4.3) was solved numerically again by DM methods. Obtained numerical results are in all cases qualitatively the same as those in [1]. Here in Fig.1 we present an example of numerical results in the case $\alpha=2 / 3 ; \omega=5 ; c_{1}=-c_{2}=15 / 8 ; \varepsilon=10^{-3}$. In calculations we chose $N=$ $11 ; M=5$; the step size $h=0.04$ for $t$, and nodes $x_{1}, x_{2}, \ldots, x_{11}$ for $x$ as zeroes of the Chebyshev polynomial $U_{11}(x)$.

\section{SOME CONCLUSIONS}

\section{Advantages:}

1. It is reasonably simple to take into account various boundary conditions, including nonlinear and nonlocal.

2. It is easily to change the number and the location of the grid points.

3. Without major changes the method can be applied to the hyperbolic equations also.

\section{Disadvantages:}

1. The increase of the number of grid points for $x$ leads to decrease of the step size $h$ for $t$.

2. The method requires considerable amount of computing time. 


\section{REFERENCES}

[1] A. Cēbers. Dynamics of an Elongated Magnetic Droplet in a Rotating Feld. Phys. Rev. E. (to be published)

[2] D. Cīrule, T.Cirulis and O. Lietuvietis. Multistep Degenerate Matrix Method for ODE. Mathematical Modelling and Analysis, 6(1), 58 - 67, 2001.

[3] T. Cirulis and O. Lietuvietis. Degenerate Matrix Method for Solving Nonlinear Systems of Differential Equations. Mathematical Modelling and Analysis, 3, 43-56, 1998.

[4] T. Cirulis and O. Lietuvietis. Degenerate Matrix Method with Chebyshev Nodes for Solving Nonlinear Systems of Differential Equations. Mathematical Modelling and Analysis, 4, $51-57,1999$.

[5] T. Cirulis and O. Lietuvietis. Degenerate Matrix Method for Solving Some Stiff Differential Equations. In: Numerical Mathematics and Advanced Applications. Proceedings of 3-rd European Conference, World Scientific, 456 - 461, 2000.

[6] E. Hairer and G. Wanner. Solving Ordinary Differential Equations 2. Springer-Verlag Berlin Heidelberg New York, 1996.

\section{DM metodo taikymai uždaviniams lygtims dalinẻmis išvestinėmis}

\section{T. Cirulis, O. Lietuvietis}

Straipsnyje nagrinèjami du išsigimusių matricų metodo taikymai uždaviniams su dalinèmis išvestinèmis spręsti. Pateikti pavyzdžiai - paprasto testinio uždavinio sprendinys ir sudètingesnio netiesinio uždavinio penktos eilès lygties dalinėmis išvestinèmis sprendinys. 